\title{
Quasi-Chiral Interactions between Quantum Emitters at the Nanoscale
}

\author{
C. A. Downing, ${ }^{1}$ J. C. López Carreño, ${ }^{2,1}$ F. P. Laussy, ${ }^{2,3}$ E. del Valle, ${ }^{1, *}$ and A. I. Fernández-Domínguez ${ }^{1, \dagger}$ \\ ${ }^{1}$ Departamento de Física Téorica de la Materia Condensada and Condensed Matter Physics Center (IFIMAC), \\ Universidad Autónoma de Madrid, E-28049 Madrid, Spain \\ ${ }^{2}$ Faculty of Science and Engineering, University of Wolverhampton, Wulfruna Street, Wolverhampton WV1 1LY, UK \\ ${ }^{3}$ Russian Quantum Center, Novaya 100, 143025 Skolkovo, Moscow Region, Russia.
}

(Dated: January 9, 2019)

\begin{abstract}
We present a combined classical and quantum electrodynamics description of the coupling between two circularly-polarized quantum emitters held above a metal surface supporting surface plasmons. Depending on their position and their natural frequency, the emitter-emitter interactions evolve from being reciprocal to nonreciprocal, which makes the system a highly tunable platform for chiral coupling at the nanoscale. By relaxing the stringent material and geometrical constraints for chirality, we explore the interplay between coherent and dissipative coupling mechanisms in the system. Thus, we reveal a quasi-chiral regime in which its quantum optical properties are governed by its subradiant state, giving rise to extremely sharp spectral features and strong photon correlations.
\end{abstract}

A quarter of a century ago, the theory of cascaded quantum systems was developed independently by Gardiner and Carmichael [1-3]. By construction, the theory describes distant source-target systems whereby non-reciprocal, unidirectional interactions arise naturally: the former is coupled to the latter while completely forbidding the opposite. Today, the emerging field of chiral quantum optics [4] seeks to realize and exploit systems exhibiting non-reciprocal light-matter interactions. Properly harnessed, the chiral coupling between quantum emitters (QEs) and photons at the quantum level promises a myriad of nontrivial applications in quantum communication and information, including non-reciprocal singlephoton devices [5], optical isolators [6], optical circulators [7] and integrated quantum optical circuits [8].

In this Letter, we present a classical and quantum electrodynamics description of the most elemental physical platform yielding non-reciprocal interactions at the nanoscale: two circularly-polarized QEs placed on top of a flat metal surface supporting tightly confined surface plasmons (SPs). The high tunability of the system, which can be manipulated through the relative position of the QEs, their natural frequencies, and the distance from the metal surface, enables us to unveil a rich landscape of coherent and dissipative emitteremitter couplings. This includes the much sought-after chiral (fully non-reciprocal) regime [4]. Through a comparison with the cascaded formalism [11], we set a well-defined criterion for chiral coupling. Moreover, by relaxing the geometric and material parameters satisfying the unidirectional conditions, we investigate the evolution of the one- and two-photon far-field spectra of the system in the transition from the conventional (reciprocal) regime. This allows us to identify a less stringent, more easily accessible quasi-chiral configuration, in which the interaction between QEs is highly directional, and where the system develops extremely sharp spectral features and strong photon correlations. Our approach does not break Lorentz reciprocity [9] since the electromagnetic (EM) fields maintain time-reversal symmetry, and it is only the emitteremitter interactions mediated by them that become unidirectional [10].
The starting point of our description for the two QEs and their EM coupling is the Hamiltonian $H=\omega_{0}\left(\sigma_{1}^{\dagger} \sigma_{1}+\sigma_{2}^{\dagger} \sigma_{2}\right)+$ $g_{12} \sigma_{1}^{\dagger} \sigma_{2}+g_{21} \sigma_{2}^{\dagger} \sigma_{1}$, where $\sigma_{i}\left(\sigma_{i}^{\dagger}\right)$ is the lowering (raising) operators of QE- $i$, and $\omega_{0}$ is its natural frequency. Hermiticity dictates that $g_{12}=g_{21}^{*}$, which makes coherent interactions fully reciprocal. We introduce damping in our model in the form of the inherent decay of both QEs and their dissipative coupling (collective decay). Both mechanisms are accounted for by the Master equation $[12,13] \partial_{t} \rho=\mathrm{i}[\rho, H]+$ $\sum_{i, j=1,2}\left(\gamma_{i j} / 2\right) \mathscr{L}_{i j} \rho+\sum_{i=1,2}\left(P_{i} / 2\right)\left(\mathscr{L}_{i i} \rho\right)^{\dagger}$, where $\gamma_{i j}$ stand for self $(i=j)$ and collective $(i \neq j)$ decay rates, and $\mathscr{L}_{i j} \rho=2 \sigma_{j} \rho \sigma_{i}^{\dagger}-\sigma_{i}^{\dagger} \sigma_{j} \rho-\rho \sigma_{i}^{\dagger} \sigma_{j}$ is the Lindblad superoperator $(\hbar=1)$. Note that we have also included the incoherent driving rates $P_{i}$, which feed population into both QEs (within the vanishing pump limit) [14]. Similar to their coherent counterparts, the collective dissipative constants satisfy $\gamma_{12}=\gamma_{21}^{*}$.

The Master equation above encompasses a rich phenomenology as a function of the coupling parameters $\left\{g_{12}, \gamma_{12}\right\}$, which are in general complex quantities. Interestingly, it maps onto the cascaded Master equation if

$$
\frac{\left|g_{12}\right|}{\left|\gamma_{12}\right|}=\frac{1}{2}, \quad \arg \left(\frac{g_{12}}{\gamma_{12}}\right)=\frac{3 \pi}{2} .
$$

The two equations above establish the magnitude and phase balance in the coherent and dissipative components of the $\mathrm{QE}$ interactions that give rise to chiral coupling. Essentially, Eqs. (1) describe the effective isolation of QE-2 from the output of QE-1. Note that the damping rates must satisfy $0 \leq\left|\gamma_{12}\right| \leq \gamma_{11}$ (from now on, we assume $\gamma_{22}=\gamma_{11}$ ), and complete equivalence to the cascaded formalism occurs only in the limit $\left|\gamma_{12}\right|=\gamma_{11}=\gamma_{22}$ [11].

We parameterize our Master equation using EM calculations for the setup sketched in the left inset of Fig. 1 (a), which mimics recent setups realizing unidirectional plasmon emission in metallic systems $[15,16]$. We model the metal response through a Drude permittivity $\epsilon(\omega)=$ $1-\omega_{\mathrm{pl}}^{2} / \omega\left(\omega+\mathrm{i} \gamma_{\mathrm{D}}\right)$, with plasma frequency $\omega_{\mathrm{pl}}=$ $3.4 \mathrm{eV}$ (which corresponds to an asymptotic SP frequency $\left.\omega_{\mathrm{sp}} \simeq \omega_{\mathrm{pl}} / \sqrt{2}=2.40 \mathrm{eV}\right)$ and investigate the impact 

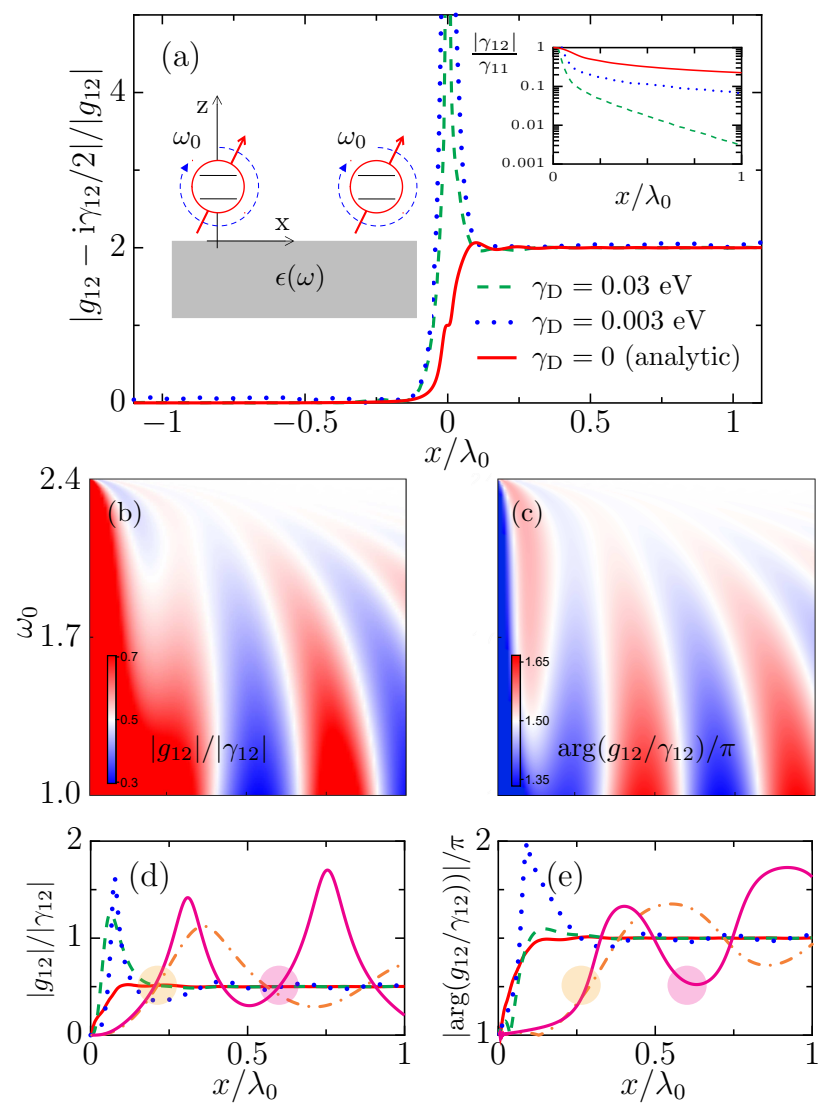

FIG. 1. (a) Effective coupling between two circularly-polarized QEs above a metal surface (left inset) versus $x / \lambda_{0}\left(z=5 \mathrm{~nm}, \omega_{0}=2.35\right.$ $\mathrm{eV})$. Right inset: dissipative coupling versus $x / \lambda_{0}$. Relative magnitude (b) and phase (c) of coherent and dissipative couplings versus $\omega_{0}$ and $x / \lambda_{0}$. Solid red lines in (d) and (e) are cuts of panels (b) and (c) at $2.35 \mathrm{eV}$. Plots for four larger $z$-values and $\gamma_{\mathrm{D}}=0.03 \mathrm{eV}$ are shown: 30 (green dashed), 60 (blue dotted), 150 (orange dot-dashed) and $300 \mathrm{~nm}$ (solid pink). Shaded circles indicate configurations satisfying $\left|g_{12}\right| /\left|\gamma_{12}\right|=1 / 2$ and $\arg \left(g_{12} / \gamma_{12}\right)=5 \pi / 4$ for the last two $z$-values.

of metal absorption by varying the Drude damping $\gamma_{\mathrm{D}}$. The dipole moment of the QEs is $\mathbf{d}=|\mathbf{d}|(\hat{x}+\mathrm{i} \hat{z}) / \sqrt{2}$, whose circularly-polarization opens up novel degrees of freedom via the phases of the coherent and dissipative coupling parameters. Within the formalism of macroscopic quantum electrodynamics [17], the coupling parameters have the form $g_{i j}=\omega_{0}^{2} \mathbf{d}_{i}^{*} \operatorname{Re}\left\{\mathbf{G}\left(\mathbf{r}_{i}, \mathbf{r}_{j}, \omega_{0}\right)\right\} \mathbf{d}_{j} / \epsilon_{0} c^{2}$ and $\gamma_{i j}=2 \omega_{0}^{2} \mathbf{d}_{i}^{*} \operatorname{Im}\left\{\mathbf{G}\left(\mathbf{r}_{i}, \mathbf{r}_{j}, \omega_{0}\right)\right\} \mathbf{d}_{j} / \epsilon_{0} c^{2}$, where $\mathbf{G}(\cdot)$ stands for the EM dyadic Green's function (DGF).

To gain insight into the emergence of chirality in our system, we compare numerical solutions of Maxwell's equations against analytical predictions for the coupling strengths obtained by keeping only the plasmon-pole contribution to the DGF $[14,18]$. From now on, we assume that both QEs are placed at the same height $z$, and introduce the position of $\mathrm{QE}-2$ relative to QE-1, $x=x_{2}-x_{1}$. In the limit of vanishing metal losses and for $k_{\mathrm{sp}}\left(\omega_{0}\right)|x| \gg 1$ (where $k_{\mathrm{sp}}\left(\omega_{0}\right)=$ $\left(\omega_{0} / c\right) \epsilon\left(\omega_{0}\right) / \sqrt{\epsilon\left(\omega_{0}\right)+1}$ is the SP wavevector at the QE fre- quency), they read

$$
g_{12}=\eta\left(\omega_{0}\right) \frac{e^{-2 \sqrt{k_{\mathrm{sp}}\left(\omega_{0}\right)^{2}-\left(\omega_{0} / c\right)^{2}} z}}{\sqrt{2 \pi \mathrm{i} k_{\mathrm{sp}}\left(\omega_{0}\right)|x|}} e^{\mathrm{i} k_{\mathrm{sp}}\left(\omega_{0}\right) x}=g_{21}^{*},
$$

and

$$
\gamma_{12}=2 \mathrm{i} \operatorname{sgn}(x) g_{12}=\gamma_{21}^{*},
$$

with $\eta(\omega)=(\omega / c)^{3}|\mathbf{d}|^{2} \epsilon(\omega)^{2} / \epsilon_{0}[\epsilon(\omega)+1]^{5 / 2}[\epsilon(\omega)-1]$. Importantly, Eqs. (2) and (3) naturally satisfy Eqs. (1), which proves that the QEs become chirally coupled when their interaction is fully mediated by confined SPs [4].

Figure 1(a) plots the absolute value of the effective coupling strength $\left|g_{12}-\mathrm{i} \gamma_{12} / 2\right|$, which combines the dissipative and coherent components, normalized to $\left|g_{12}\right|$, as a function of the distance between the two QEs $\left(\omega_{0}=2.35 \mathrm{eV}, \lambda_{0}=527\right.$ $\mathrm{nm}$, and $z=5 \mathrm{~nm}$ ). Numerical calculations for two different $\gamma_{\mathrm{D}}$ (green dashed and blue dotted lines) are compared against the lossless analytical result (red solid line). Remarkably, for $|x| \gtrsim 0.1 \lambda_{0} \simeq 53 \mathrm{~nm}$, the QE interactions are governed by SPs (analytical and numerical predictions are in perfect agreement) and the effective coupling becomes directional: its magnitude approaches $2\left|g_{12}\right|$ for $\operatorname{sgn}(x)>0$ and vanishes for $\operatorname{sgn}(x)<0$. We also observe that the inclusion of metal absorption does not degrade the chiral character of the QE coupling, which is insensitive to $\gamma_{D}$. The right inset shows that the only effect of the Drude losses is to reduce the efficiency of the dissipative coupling mechanism, weighted by $\left|\gamma_{12}\right| / \gamma_{11}$. Notably, recent reports have demonstrated that this ratio (termed the $\beta$-factor) can be optimized in complex plasmonic structures $[19,20]$ beyond our proof-of-principle proposal.

Figure $1(b, c)$ analyze the deviation of SP-assisted coupling from Eqs. (1) as a function of the $\mathrm{QE}$ distance and natural frequency $(z=5 \mathrm{~nm})$. We observe that both the magnitude ratio (b) and phase difference (c) undergo oscillations around the chiral condition (white regions), which become stronger as $x / \lambda_{0}$ and $\omega_{0}$ decrease. Both maps are obtained from analytical expressions [14], and demonstrate the possibility of varying the degree of chirality in QE coupling exclusively through SPs. Red solid lines in Figs. 1(d) and (e) plot cuts at $\omega_{0}=2.35 \mathrm{eV}$ in panels (b) and (c), respectively. Exact numerical calculations for $\gamma_{\mathrm{D}}=0.03 \mathrm{eV}$ and increasing $z$ are shown in dashed green $(30 \mathrm{~nm})$, dotted blue $(60 \mathrm{~nm})$, dotdashed orange $(150 \mathrm{~nm})$, and thin pink $(300 \mathrm{~nm})$ lines. The deviations from Eqs. (1) are larger than in the upper panels, as a result of the damping experienced by SPs in their propagation. This demonstrates that the tunability of the system increases further when the contribution of free-propagating modes to the DGF becomes comparable to SPs.

Now we have proven the tunability of emitter-emitter interactions in our EM set-up, we investigate its influence on quantum optical properties. Figure 2(a) reveals the hallmark of chirality in the QE populations. It shows the steady-state population of QE-2, $n_{2}=\left\langle\sigma_{2}^{\dagger} \sigma_{2}\right\rangle$, normalized to its population in isolation, $n_{0}=P_{2} /\left(P_{2}+\gamma_{22}\right)$, versus $\left|g_{12}\right| /\left|\gamma_{12}\right|$ and $\arg \left(g_{12} / \gamma_{12}\right)$. From now on, and unless stated otherwise, we 

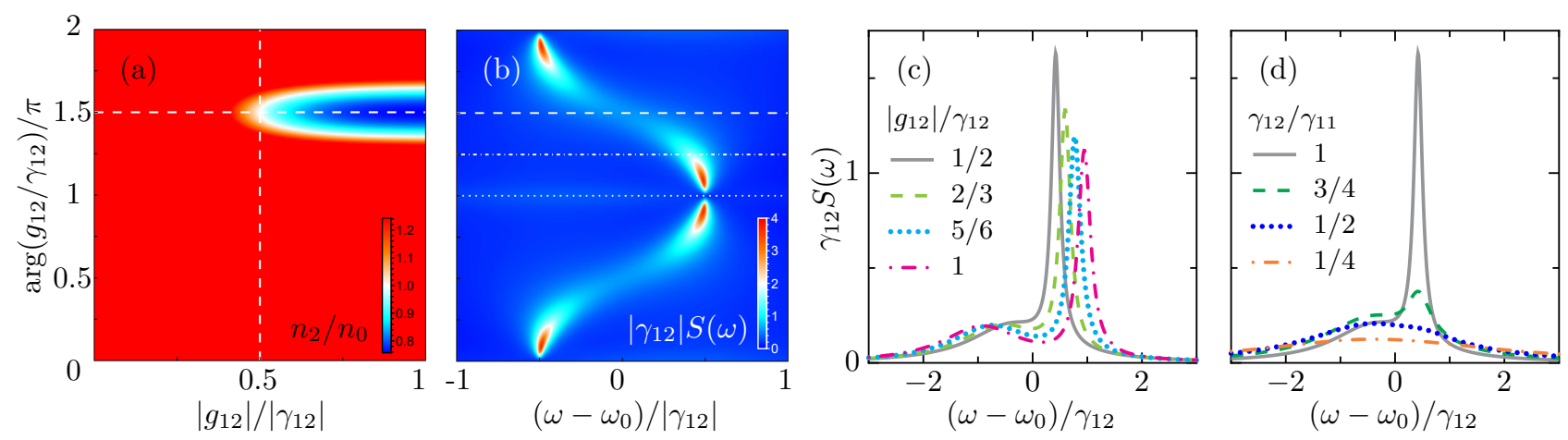

FIG. 2. (a) Steady-state population of QE-2, normalized to its population in isolation, as a function of the relative magnitude and phase of the coherent and dissipative couplings. White dashed lines plot Eqs. (1). (b) Far-field spectrum $S(\omega)$ as a function of arg $\left(g_{12} / \gamma_{12}\right)$ for $\left|g_{12}\right|=\left|\gamma_{12}\right| / 2\left(P_{1}=P_{2}=\gamma_{11} / 100\right.$ and $\left.\left|\gamma_{12}\right|=\gamma_{11}\right)$. The three horizontal lines indicate the configurations in Fig. 3. (c) $S(\omega)$ for increasing ratios of $\left|g_{12}\right| / \gamma_{11}$, with $\arg \left(g_{12} / \gamma_{12}\right)=5 \pi / 4$ and $\gamma_{12}=\gamma_{11}$. (d) $S(\omega)$ for decreasing $\left|\gamma_{12}\right| / \gamma_{11}$, with arg $\left(g_{12} / \gamma_{12}\right)=5 \pi / 4$, $\left|g_{12}\right|=\left|\gamma_{12}\right| / 2$. The grey lines in (c) and (d) are evaluated along the white dot-dashed (middle) line in panel (b).

take $\gamma_{12}=\left|\gamma_{12}\right|=\gamma_{11}$. As anticipated, when Eqs. (1) are fulfilled (intersection of the horizontal and vertical long-dashed white lines) the backaction is eliminated (the output of QE2 drives QE-1 but the opposite is prevented) and one indeed finds $n_{2}=n_{0}$. This is a manifestation of chiral coupling and is realizable in the proposed emitter-emitter setup due to its nontrivial field configurations. We note that $n_{2}=n_{0}$ along the white ring in Fig. 2(a), which always includes the chiral point, however its aspect ratio depends strongly on the system parameters. Inside (outside) this ring, we find $n_{2} / n_{0}$ smaller (larger) than unity.

We focus now on the parameter range given by the vertical white dashed line in Fig. 2(a), and investigate the properties of the coupled QEs when only the magnitude condition for chirality is met. As shown in Fig. 1, this regime is significantly more accessible than the chiral configuration, as the geometric (QEs height and separation) and material (QE natural frequency) constraints on the system are greatly relaxed. We explore the system through the normalized power spectrum [21] $S(\omega)=\lim _{t \rightarrow \infty} \operatorname{Re}\left\{\int_{0}^{\infty}\left\langle\xi^{\dagger}(t) \xi(t+\right.\right.$ $\left.\tau)\rangle e^{\mathrm{i} \omega \tau} \mathrm{d} \tau\right\} /\left(\pi n_{\xi}\right)$, which accounts for coherent superposition of the photon emission by both QEs, with $\xi=\left(\sigma_{1}+\right.$ $\left.\sigma_{2}\right) / \sqrt{2}$ and $n_{\xi}=\left\langle\xi^{\dagger} \xi\right\rangle$. Figure 2(b) plots $S(\omega)$ versus $\arg \left(g_{12} / \gamma_{12}\right)$ for $\left|g_{12}\right|=\left|\gamma_{12}\right| / 2$. It reveals that, for certain phase differences, an extremely narrow peak emerges that completely dominates the spectral properties of the system. We term the configuration yielding this sharp spectral feature as quasi-chiral, which smoothly evolves into the drastically lower and broader single-peaked spectrum characteristic of the reciprocal $\left[\arg \left(g_{12} / \gamma_{12}\right)=0, \pi, 2 \pi\right]$ and chiral $\left[\arg \left(g_{12} / \gamma_{12}\right)=\pi / 2,3 \pi / 2\right]$ regimes. Figures $2(\mathrm{c})$ and (d) analyze the sensitivity of the quasi-chiral peak to $\left|g_{12}\right| /\left|\gamma_{12}\right|$ and $\left|\gamma_{12}\right| / \gamma_{11}$, respectively. The grey solid spectrum in both panels is a cut along the white dot-dashed line in panel (b) $\left[\arg \left(g_{12} / \gamma_{12}\right)=5 \pi / 4\right]$. Upon increasing the EM coupling strength, and thus away from the chiral condition, we find a remarkable robustness in the spectrum, which still presents a (slightly blue-shifted) prominent peak for $\left|g_{12}\right|=\left|\gamma_{12}\right|$ [panel (c)]. Contrarily, by decreasing the dissipative coupling $S(\omega)$ flattens, which indicates the crucial role the $\beta$-factor plays in the emergence of the quasi-chiral phenomenology [panel (d)].

We analyze in more detail the emission spectrum of the two coupled QEs. For that purpose, we study the three different EM coupling configurations marked by horizontal lines in

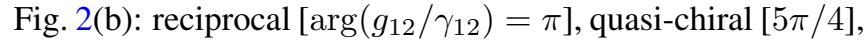
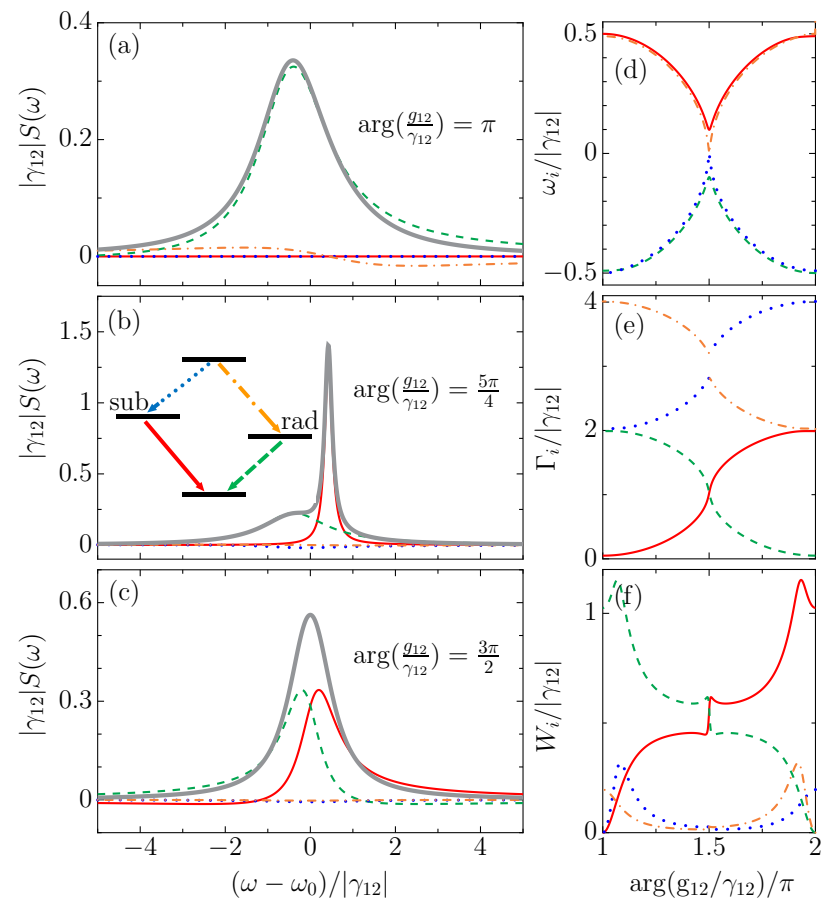

FIG. 3. Left panels: $S(\omega)$ (thick gray lines) and its constituents, $S_{i}(\omega)$ (thin colored lines), for the three system configurations indicated by horizontal white lines in Fig. 2(b): reciprocal (a), quasichiral (b), and chiral (c). Right panels: spectral position (d), width (e) and weight (f) of $S_{i}(\omega)$ as a function of $\arg \left(g_{12} / \gamma_{12}\right)$. In all panels, $P_{1}=P_{2}=\gamma_{11} / 100,\left|g_{12}\right|=\left|\gamma_{12}\right| / 2$, and $\gamma_{12} \simeq \gamma_{11}$. 
and chiral $[3 \pi / 2]$. The quasi-chiral condition is fulfilled at the two shaded circles in Fig. 1(d-e), in which the interaction between the QEs is highly directional, although not fully unidirectional [14]. The emission spectra for the reciprocal, quasi-chiral and chiral regimes are shown as the solid grey lines in Fig. 3(a), (b) and (c), respectively (note the different vertical scales). The panels also display the decomposition $S(\omega)=\sum_{i=1}^{4} S_{i}(\omega)[12,14]$, where the contributions $S_{i}(\omega)$ (non-solid color lines) arise from the four transitions that can take place in the system. They are illustrated by the diamond level scheme in the inset of Fig. 3(b), with vertices at frequencies $\left\{0, \omega_{\text {rad }}, \omega_{\text {sub }}, 2 \omega_{0}\right\}$. Note that $\omega_{\text {rad }}$ and $\omega_{\text {sub }}$ result from the diagonalization of the Liouvillian $\mathscr{L}$ and, therefore, vary with the parameters. We observe that the reciprocally coupled spectrum [panel (a)] is governed by a single peak centered at $\omega_{\text {rad }} \simeq \omega_{0}-\left|g_{12}\right|=\omega_{0}-\left|\gamma_{12}\right| / 2$ which originates from the transition from the radiant to the ground state (green dashed line). Note that $\arg \left(g_{12} / \gamma_{12}\right)=\pi$ in this case, which yields $\omega_{\mathrm{rad}}<\omega_{0}$ [14]. The chiral system [panel (c)] presents a single, broader maximum which emerges due to the spectral overlapping of the emission of the two single-excitation states $\left(\omega_{\text {rad }}=\omega_{\text {sub }}=\omega_{0}\right)$. The two QEs are only weakly coupled and $S(\omega)$ resembles the single QE spectrum. Finally, we find that, surprisingly, the sharp peak in the quasi-chiral emission [panel (b)] originates from the decay of the subradiant state, with $\omega_{\text {sub }} \simeq \omega_{0}+0.4\left|\gamma_{12}\right|$. Note that a second, much lower and broader maximum occurs at $\omega_{\mathrm{rad}} \simeq \omega_{0}-0.4\left|\gamma_{12}\right|$.

To examine the causes of the evolution of the emission spectrum with $\arg \left(g_{12} / \gamma_{12}\right)$, we plot in the right column of Fig. 3 the resonant frequencies $\omega_{i}(\mathrm{~d})$, the decay rates $\Gamma_{i}$ (e), and the weights $W_{i}$ (f) for the four $S_{i}(\omega)$ contributions $[12,14]$. We can observe that by varying the phase difference between coherent and dissipative couplings from the reciprocal to the chiral configuration, the frequency and decay rate of the radiant and subradiant states merge into the single QE values: $\omega_{0}$ and $\gamma_{12}=\gamma_{11}$. In the evolution, the weight of the subradiant contribution increases faster than its linewidth, which gives rise to the formation, narrowing and blue-shifting of the quasi-chiral spectral maximum shown in Fig. 2(b). In particular, Fig. 3(e) evidences that the linewidth of the sub-radiant contribution is much smaller than $\gamma_{12}$ at $\arg \left(g_{12} / \gamma_{12}\right)=5 \pi / 4$, which gives rise to the sharp spectral peak in the quasi-chiral spectrum.

In order to access the emission dynamics of the coupled QEs at a deeper level, we investigate the two-photon correlations in frequency, via the theory of two-photon spectra [22]. Using the formalism of Ref. [23], we compute the two-photon spectrum $g_{\Omega}^{(2)}\left(\omega_{\mathrm{I}}, \omega_{\mathrm{II}}\right)$ of our system for photons detected at frequencies $\omega_{\text {I,II }}$ (for more details, see Sec. 3 of Ref. [14]). We assume zero time delay and access the correlations via detectors with spectral width $\Omega=\left|\gamma_{12}\right| / 5$. We plot in Fig. 4 (a, b, c) spectra for the same $\arg \left(g_{12} / \gamma_{12}\right)$ as in Fig. 3 (a, b, c). In the three panels, a dashed thick line marks the subradiant peak position and the dot-dashed thin one marks the radiant frequency. The reciprocal system (a) broadly demonstrates
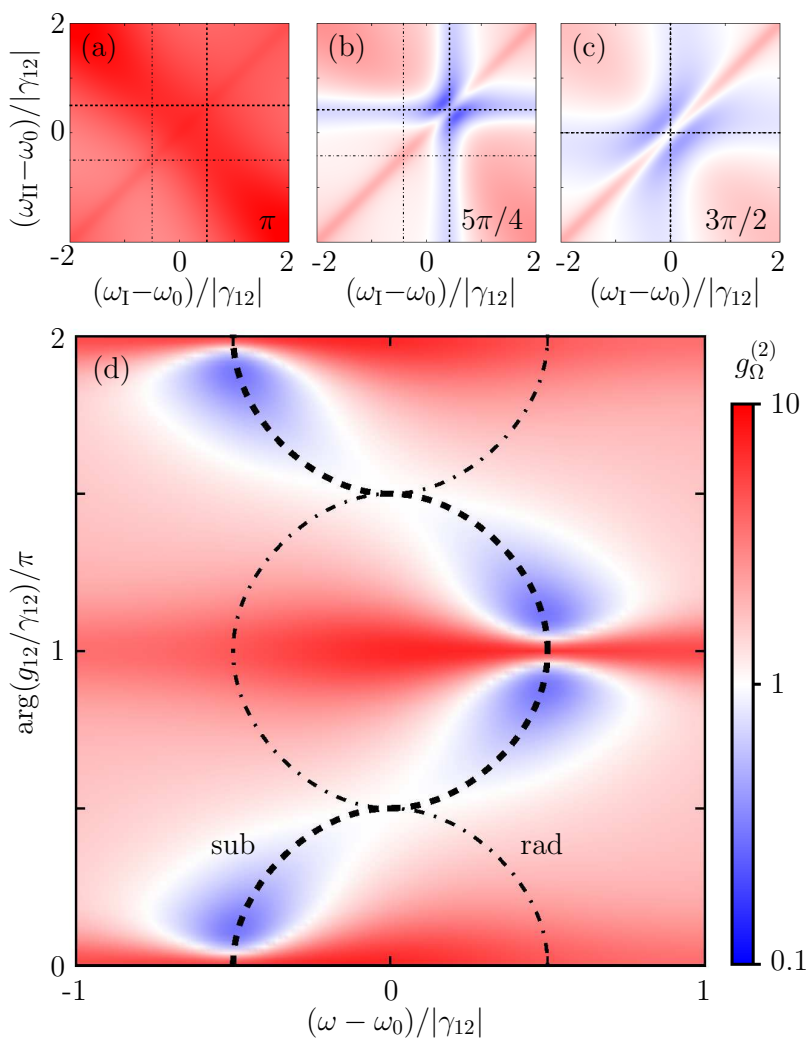

FIG. 4. Two-photon spectra at zero time delay, $g_{\Omega}^{(2)}$, as a function of the emission frequencies $\omega_{\text {I }}$ and $\omega_{\text {II }}$. The three cases in Fig. 3 are shown: (a) reciprocal, (b) quasi-chiral, and (c) chiral. The system is resonantly excited with a laser, with filter size $\Omega=\left|\gamma_{12}\right| / 5$. (d) Autocorrelation function, $g_{\Omega}^{(2)}\left(\omega_{\mathrm{I}}=\omega_{\mathrm{II}}=\omega\right)$, versus $\arg \left(g_{12} / \gamma_{12}\right)$. In all panels, the thick dashed (thin dot-dashed) lines track the (sub)radiant state.

bunching [red regions, $g_{\Omega}^{(2)}>1$ ], with little fine structure. However, upon evolving through the quasi-chiral configuration (b) towards the chiral limit (c), a remarkable butterfly structure appears: two bands of antibunching [blue regions, $\left.g_{\Omega}^{(2)}<1\right]$ pierced by a diagonal line of higher values of $g_{\Omega}^{(2)}$. This is the typical manifestation of a single-photon emission, in this case at the sub-radiant frequency [23]. In panel (d), we map the autocorrelation function $g_{\Omega}^{(2)}\left(\omega_{\mathrm{I}}=\omega_{\mathrm{II}}=\omega\right)$ versus the phase difference between coherent and dissipative coupling strengths. The close resemblance to the one-photon spectrum of Fig. 2(b) is immediately apparent. We conclude by inspection that the minimum of $g_{\Omega}^{(2)}$ overlaps almost exactly with the subradiant peak position. Most notably, the global minimum in the correlation function does not occur at the chiral conditions but when the two QEs are quasi-chirally coupled, further highlighting the singular optical properties that emerge in the quasi-chiral regime.

To conclude, we have investigated the emergence of chirality in the interactions between two quantum emitters. Exploring the interplay between coherent and dissipative mechanisms, we find naturally the non-reciprocal coupling configu- 
ration, which enables us to identify the physical conditions for its occurrence. Through analytical and numerical EM calculations, we have shown that tunable chiral interactions can be realized in a nanoscale platform consisting of two circularlypolarized emitters held above a metal surface. Finally, we have unveiled the rich quantum optical properties of the quasichiral regime, in which the conditions for non-reciprocity are only partially met. We have shown that the subradiant state of the system governs its one- and two-photon spectra, which, remarkably, gives rise to both intense emission and strong photon correlations within the same spectral window. Our findings set the theoretical grounds and provide guidance towards the development and optimization of quantum optical functionalities associated with the fine tuning between coherent and dissipative light-matter interactions, beyond the highly stringent chiral regime.

This work has been funded by the Spanish MINECO under contract FIS2015-64951-R (CLAQUE) and through the 'María de Maeztu' programme for Units of Excellence in R\&D (MDM-2014-0377). AIFD acknowledges funding from the EU Seventh Framework Programme under Grant Agreement FP7-PEOPLE-2013-CIG-630996.

* elena.delvalle@uam.es

† a.fernandez-dominguez@uam.es

[1] C. W. Gardiner, Driving a quantum system with the output field from another driven quantum system, Phys. Rev. Lett. 70, 2269 (1993).

[2] H. J. Carmichael, Quantum trajectory theory for cascaded open systems, Phys. Rev. Lett. 70, 2273 (1993).

[3] C. Gardiner and P. Zoller, Quantum Noise (Springer, Berlin, 2004).

[4] P. Lodahl, S. Mahmoodian, S. Stobbe, A. Rauschenbeutel, P. Schneeweiss, J. Volz, H. Pichler and P. Zoller, Chiral quantum optics, Nature 541, 473 (2017).

[5] I. Shomroni, S. Rosenblum, Y. Lovsky, O. Bechler, G. Guendelman, and B. Dayan, All —optical routing of single photons by a one-atom switch controlled by a single photon, Science $\mathbf{3 4 5}$, 903 (2014).

[6] C. Sayrin, C. Junge, R. Mitsch, B. Albrecht, D. O'Shea, P. Schneeweiss, J. Volz, and A. Rauschenbeutel, Nanophotonic optical isolator controlled by the internal state of cold atoms, Phys. Rev. X 5, 041036 (2015).

[7] I. Sollner, S. Mahmoodian, S. L. Hansen, L. Midolo, A. Javadi, G. Kirsanske, T. Pregnolato, H. El-Ella, E. H. Lee, J. D. Song, S. Stobbe and P. Lodahl, Deterministic photon-emitter coupling in chiral photonic circuits, Nature Nanotechnology $\mathbf{1 0}$, 775 (2015).

[8] S. Barik, A. Karasahin, C. Flower, T. Cai, H. Miyake, W. DeGottardi, M. Hafezi, and E. Waks, A topological quantum optics interface, Science 359, 666 (2018).

[9] C. Caloz, A. Alù, S. Tretyakov, D. Sounas, K. Achouri, and Z.-L. Deck-Léger, Electromagnetic nonreciprocity, Phys. Rev. Applied 10, 047001 (2018).

[10] S. Fan, R. Baets, A. Petrov, Z. Yu, J. D. Joannopoulos, W. Freude, A. Melloni, M. Popovic, M. Vanwolleghem, D. Jalas, M. Eich, M. Krause1, H. Renner, E. Brinkmeyer,
C. R. Doerr, Comment on "Nonreciprocal light propagation in a silicon photonic circuit", Science 335, 38 (2012).

[11] A. Metelmann and A. A. Clerk, Nonreciprocal photon transmission and amplification via reservoir engineering, Phys. Rev. X 5, 021025 (2015).

[12] E. del Valle, Strong and weak coupling of two coupled qubits, Phys. Rev. A. 81, 053811 (2010).

[13] D. Martin-Cano, A. Gonzalez-Tudela, L. Martin-Moreno, F. J. Garcia-Vidal, C. Tejedor, and E. Moreno, Dissipationdriven generation of two-qubit entanglement mediated by plasmonic waveguides, Phys. Rev. B 84, 235306 (2011).

[14] See the Supplemental Material, which includes Refs. [24-37], for further details and derivations.

[15] F. J. Rodríguez-Fortuño, G. Marino, P. Ginzburg, D. O'Connor, A. Martínez, G. A. Wurtz, and A. V. Zayats, Near-field interference for the unidirectional excitation of electromagnetic guided modes, Science 340, 328 (2013).

[16] D. O'Connor, P. Ginzburg, F. J. Rodríguez-Fortuño, G. A. Wurtz, and A. V. Zayats, Spin-orbit coupling in surface plasmon scattering by nanostructures, Nat. Comm. 5, 5327 (2014).

[17] H. T. Dung, L. Knoll, and D.-G. Welsch, Resonant dipoledipole interaction in the presence of dispersing and absorbing surroundings, Phys. Rev. A 66, 063810 (2002).

[18] A. Yu. Nikitin, S, G. Rodrigo, F. J. Garcia-Vidal and L. MartinMoreno, In the diffraction shadow: Norton waves versus surface plasmon polaritons in the optical region New J. Phys. 11, 123020 (2009).

[19] E. Bermúdez-Ureña, C. Gonzalez-Ballestero, M. Geiselmann, R. Marty, I. P. Radko, T. Holmgaard, Y. Alaverdyan, E. Moreno, F. J. Garcia-Vidal, S. I. Bozhevolnyi and R. Quidant, Coupling of individual quantum emitters to channel plasmons, Nature Comm. 6, 7883 (2015).

[20] A. V. Akimov, A. Mukherjee, C. L. Yu, D. E. Chang, A. S. Zibrov, P. R. Hemmer, H. Park, and M. D. Lukin, Generation of single optical plasmons in metallic nanowires coupled to quantum dots, Nature 450, 402 (2007).

[21] J. C. López Carreño, C. Sánchez Muñoz, E. del Valle and F. P. Laussy, Excitation with quantum light. II. Exciting a twolevel system Phys. Rev. A 94, 063826 (2016).

[22] E. del Valle, A. Gonzalez-Tudela, F. P. Laussy, C. Tejedor, and M. J. Hartmann, Theory of frequency-filtered and time-resolved N-photon correlations, Phys. Rev. Lett. 109, 183601 (2012).

[23] A. Gonzalez-Tudela, F. P. Laussy, C. Tejedor, M. J. Hartmann and E. del Valle, Two-photon spectra of quantum emitters, New J. Phys. 15, 033036 (2013).

[24] J. C. López Carreño, E. del Valle, and F. P Laussy, Frequencyresolved Monte Carlo, Sci Rep. 8, 6975 (2018).

[25] F. P. Laussy, E. del Valle, and C. Tejedor, Luminescence spectra of quantum dots in microcavities. I. Bosons, Phys. Rev. B 79, 235325 (2009).

[26] E. del Valle, Microcavity Quantum Electrodynamics (VDM Verlag, 2010).

[27] E. del Valle and F. P. Laussy, Regimes of strong light-matter coupling under incoherent excitation, Phys. Rev. A 84, 043816 (2011).

[28] L. Novotny and B. Hecht, Principles of Nano-Optics (Cambridge University Press, Cambridge, 2006).

[29] A. Yu. Nikitin, F. J. Garcia-Vidal, and L. Martin-Moreno, Analytical expressions for the electromagnetic dyadic Green's function in graphene and thin layers, IEEE Journal of Selected Topics in Quantum Electronics 19, 4600611 (2013).

[30] A. L. Falk, F. H. L. Koppens, C. L. Yu, K. Kang, N. de Leon Snapp, A. V. Akimov, M.-H. Jo, M. D. Lukin and H. Park, Near-field electrical detection of optical plasmons and 
single-plasmon sources, Nat. Phys. 5, 475 (2009).

[31] B. Le Feber, N. Rotenberg, and L. Kuipers, Nanophotonic control of circular dipole emission, Nat. Commun. 6, 6695 (2015).

[32] R. J. Coles, D. M. Price, J. E. Dixon, B. Royall, E. Clarke, P. Kok, M. S. Skolnick, A. M. Fox and M. N. Makhonin, Chirality of nanophotonic waveguide with embedded quantum emitter for unidirectional spin transfer, Nat. Commun. 7, 11183 (2016).

[33] For a review of chirality at the photon-molecule level, see D. L. Andrews, Quantum formulation for nanoscale optical and material chirality: symmetry issues, space and time parity, and observables, J. Opt. 20, 033003 (2018).

[34] E. Moreno, S. G. Rodrigo, S. I. Bozhevolnyi, L. MartínMoreno, and F. J. García-Vidal, Guiding and focusing of elec- tromagnetic fields with wedge plasmon polaritons, Phys. Rev. Lett. 100, 023901 (2008).

[35] D. E. Chang, A. S. Sorensen, P. R. Hemmer, and M. D. Lukin, Quantum optics with surface plasmons, Phys. Rev. Lett. 97, 053002 (2006).

[36] N. Rivera, I. Kaminer1, B. Zhen, J. D. Joannopoulos, and M. Soljacic, Shrinking light to allow forbidden transitions on the atomic scale, Science 353, 263 (2016).

[37] L. Martín-Moreno, F. J. García de Abajo, and F. J. GarcíaVidal, Ultraefficient coupling of a quantum emitter to the tunable guided plasmons of a carbon nanotube, Phys. Rev. Lett. 115, 173601 (2015). 